\title{
eNeuro
}

Research Article: New Research / Cognition and Behavior

\section{Theta-gamma cross-frequency transcranial alternating current stimulation over the trough impairs cognitive control}

https://doi.org/10.1523/ENEURO.0126-20.2020

Cite as: eNeuro 2020; 10.1523/ENEURO.0126-20.2020

Received: 2 April 2020

Revised: 15 July 2020

Accepted: 15 July 2020

This Early Release article has been peer-reviewed and accepted, but has not been through the composition and copyediting processes. The final version may differ slightly in style or formatting and will contain links to any extended data.

Alerts: Sign up at www.eneuro.org/alerts to receive customized email alerts when the fully formatted version of this article is published.

Copyright $(2020$ Turi et al.

This is an open-access article distributed under the terms of the Creative Commons Attribution 4.0 International license, which permits unrestricted use, distribution and reproduction in any medium provided that the original work is properly attributed. 
1 1. Manuscript title: Theta-gamma cross-frequency transcranial alternating current stimulation

2 over the trough impairs cognitive control

3 2. Abbreviated title (50 character max): Theta-gamma tACS alters cognitive control

4 3. List of authors and affiliations: Zsolt Turi ${ }^{1,2 *}$, Matthias Mittner ${ }^{3 *}$, Albert Lehr $^{1}$, Hannah

5 Bürger ${ }^{1}$, Andrea Antal $^{1}$, and Walter Paulus ${ }^{1}$

$6 \quad$ *Equal contribution

7 'Department of Clinical Neurophysiology, University Medical Center Göttingen, Göttingen 37073,

8 Germany

$9{ }^{2}$ Department of Neuroanatomy, Institute of Anatomy and Cell Biology, Faculty of Medicine,

10 University of Freiburg, Freiburg, Germany.

$11{ }^{3}$ Department of Psychology, UiT The Arctic University of Norway

12

13

\section{Author contributions}

ZT: conceptualization, study design, project administration, methodology, software (behavioral paradigm), supervised data collection, supervised medical student, prepared illustrations, data visualization, interpreted data, data curation, wrote original draft and revised manuscript.

MM: formal statistical analysis, data visualization, computational modelling of behavioral data, interpreted data, data curation, wrote original draft and revised manuscript.

AL: contributed to formal analysis, contributed to preparing illustrations, data visualization, interpreted data and wrote original draft and revised manuscript.

HB: data collection (as part of her medical dissertation at the University Medical Center Göttingen, Germany, supervised by author AA), transcribed data, contributed to writing original draft and revised manuscript. 
AA: project administration, supervised medical student, contributed to writing original draft and revised manuscript.

WP: study design, resources and funding acquisition, contributed to writing original draft and revised manuscript.

5. Correspondence should be addressed to Zsolt Turi (zsoltturi@gmail.com) and Matthias Mittner (matthias.mittner@uit.no)

6. Number of Figures : 5

7. Number of Tables: 1

8. Number of Multimedia: 1

9. Number of words for Abstract: 247

10. Number of words for Significance Statement: 76

11. Number for words for Introduction: 750

12. Number of words for Discussion: 1580

13. Acknowledgements: The authors wish to thank Dr. med. Anja Manig, Dr. med. Sebastian Schade, Dr. med. Dirk Czesnik and Dr. med. Claire Halsband for the neurological examinations. We thank Prof. Thomas Crozier for his comments on the manuscript.

14. Conflict of interest: Authors report no conflict of interest.

15. Funding sources: This research was financially supported by DFG PA 419/15-1 awarded to WP. AL was supported by the IMPRS Neurosciences. AA was supported by the State of Lower Saxony, Germany (76251-12-7/19 (ZN 3456))

16. Link to repository: https://github.com/ihrke/2020 cfc tacs 

stimulation over the trough impairs cognitive control

47

Zsolt Turi $^{1,2 *}$, Matthias Mittner ${ }^{3 *}$, Albert Lehr $^{1}$, Hannah Bürger $^{1}$, Andrea Antal $^{1}$, and Walter Paulus ${ }^{1}$

${ }^{*}$ Equal contribution

${ }^{1}$ Department of Clinical Neurophysiology, University Medical Center Göttingen,

52 Göttingen 37073, Germany

$53 \quad{ }^{2}$ Department of Neuroanatomy, Institute of Anatomy and Cell Biology, Faculty of 54 Medicine, University of Freiburg, Freiburg, Germany.

$55{ }^{3}$ Department of Psychology, UiT The Arctic University of Norway

57 Correspondence should be addressed to Zsolt Turi (zsoltturi@gmail.com) and Matthias 58 Mittner (matthias.mittner@uit.no) 


\section{Abstract}

Cognitive control is a mental process, which underlies adaptive goal-directed decisions. Previous studies have linked cognitive control to electrophysiological fluctuations in the theta band and theta-gamma cross-frequency coupling (CFC) arising from the cingulate and frontal cortices. Yet, to date the behavioral consequences of different forms of thetagamma CFC remain elusive. Here, we studied the behavioral effects of the theta-gamma CFC via transcranial alternating current stimulation (tACS) designed to stimulate the frontal and cingulate cortices in humans. Using a double-blind, randomized, repeated measures study design, 24 healthy participants were subjected to three active and one control CFC-tACS conditions. In the active conditions, $80 \mathrm{~Hz}$ gamma tACS was coupled to $4 \mathrm{~Hz}$ theta tACS. Specifically, in two of the active conditions, short gamma bursts were coupled to the delivered theta cycle to coincide with either its peaks or troughs. In the third active condition, the phase of a theta cycle modulated the amplitude of the gamma oscillation. In the fourth, control protocol, $80 \mathrm{~Hz}$ tACS was continuously superimposed over the $4 \mathrm{~Hz}$ tACS, therefore lacking any phase-specificity in the CFC. During the 20-minute of stimulation, the participants performed a Go/NoGo monetary reward- and punishment-based instrumental learning task. A Bayesian hierarchical logistic regression analysis revealed that relative to the control, the peak-coupled tACS had no effects on the behavioral performance, whereas the trough-coupled tACS and, to a lesser extent, amplitude-modulated tACS reduced performance in conflicting trials. Our results suggest that cognitive control depends on the phase-specificity of the thetagamma CFC. 

81 Statement of significance
82 This study investigated the behavioral effects of different forms of theta-gamma cross-
83 frequency coupling in cognitive control. To this aim, we delivered cross-frequency
84 transcranial alternating current stimulation over the cingulate and frontal cortices in
85 humans. We found that when gamma tACS was coupled to the trough of theta tACS, the
86 stimulation worsened the ability of healthy participants to employ cognitive control. Our
87 findings highlight the role of theta-gamma cross frequency coupling in complex goal-
88 directed behavior in humans. 


\section{Introduction}

In goal-directed behavior, contextual and reward-related information should be effectively linked to form action plans in order to accomplish goals and perform decisions in a flexible and prospective manner (Helfrich \& Knight, 2019). In humans, at least three main behavioral control systems influence the decisions: The Pavlovian system, the model-free and the model-based instrumental systems (Guitart-Masip et al., 2014). The Pavlovian system is responsible for automatic, reflexive response tendencies that depend on the valence of the stimulus. It facilitates approaching behavior for rewarding stimuli and response inhibition for unrewarding ones (Guitart-Masip et al., 2014). The model-free system gradually incorporates the behavioral consequences of actions by computing the difference between the predicted and received outcome. The modelbased system creates an internal world model, which enables flexible, prospective planning. Therefore, decisions do not exclusively rely on the outcome history (Helfrich \& Knight, 2019).

Conflict can arise between the Pavlovian and instrumental behavioral control systems, when the evolutionary hard-wired, valence-response associations do not support adaptive behavior. This situation occurs when approaching rewards is maladaptive, or when rewards can be secured by response inhibition rather than by approach (Guitart-Masip et al., 2012). Cognitive control is a mental process for resolving this conflict between the behavioral control systems (Guitart-Masip et al., 2014; Shenhav et al., 2017).

The oscillatory activity in the theta and gamma frequency bands and their interaction may play a crucial role in cognitive control (Cavanagh \& Frank, 2014; Cohen, 2014). Theta-gamma, phase-amplitude cross-frequency coupling (CFC) is one form of such 
interaction, where the phase of the theta oscillation modulates the amplitude of the gamma oscillation (Canolty \& Knight, 2010). Human intracranial electrophysiological recordings revealed that theta-gamma, phase-amplitude CFC in the anterior cingulate cortex (ACC) and dorsolateral prefrontal cortex (DLPFC) emerges during cognitive control (Smith et al., 2015). E.g., Smith and colleagues found that the amplitude of the high gamma oscillation was highest in a specific phase range of the theta oscillation (ca. $0^{\circ}-60^{\circ}$ ) during a cognitive control task (Smith et al., 2015).

To study how participants learn to overcome the Pavlovian bias by utilizing cognitive control mechanisms, we used a probabilistic Go/NoGo instrumental learning task (Cavanagh et al., 2013). We tested the behavioral relevance of theta-gamma crossfrequency coupling in humans via transcranial alternating current stimulation (tACS), which can externally generate oscillating electric fields in the brain (Peterchev et al., 2012). We utilized three CFC-tACS protocols delivered in the theta and gamma frequency bands: Peak- and trough-coupled tACS and amplitude-modulated tACS (Alekseichuk et al., 2016; Amador de Lara et al., 2017; Minami \& Amano, 2017). In the context of the present study, the notion of peak and trough refers to the local maximum and minimum of the amplitude of the delivered theta tACS wave, to which the short gamma tACS burst was coupled. In the amplitude-modulated protocol, the amplitude of the gamma oscillation was modulated by the phase of the theta wave.

We hypothesized that the peak-coupled tACS would improve the accuracy and/or the speed of learning relative to the control stimulation. We based this hypothesis on the notion that these protocols mimic the phase-specificity of theta-gamma CFC when signaling the need for cognitive control (Smith et al., 2015). Moreover, we also anticipated that the trough-coupled tACS would impair behavioral performance because 
this pattern is contrary to that activity naturally occurring during the successful implementation of cognitive control (Smith et al., 2015). Third, we expected that modulating the CFC between the ACC and DLPFC via CFC-tACS protocols should affect the amount of Pavlovian bias. In particular, facilitating the CFC between the ACC and DLPFC via the peak-coupled tACS would be thought to increase the efficacy of the ACC to signal the need for cognitive control and thereby increase the degree of modelbased control implemented by the DLPFC (Smith et al., 2015). This, in turn, might lead to a decreased amount of Pavlovian bias. On the other hand, disrupting the CFC between the ACC and the DLPFC via the trough-coupled tACS should decrease the efficacy of signaling the need for cognitive control. This may impair the efficacy of implementing model-based control and therefore lead to a higher degree of Pavlovian bias. Fourth, we expected that amplitude-modulated tACS would improve behavioral performance by entraining the ongoing theta oscillation by the envelope of the high frequency stimulation (Negahbani et al., 2018). The amplitude-modulated tACS protocol would increase the theta synchrony in the cingulate and frontal cortices (Negahbani et al., 2018), which in turn would improve the ability of the participants to apply cognitive control.

\section{Methods}

\subsection{Participants}

Twenty-four healthy, native German-speaking adult volunteers (12 female, mean age \pm SD: $23.0 \pm 3.26$ years, age range from 18 to 30 years) joined the study. This number of participants was chosen to allow a complete randomization of the order of the four tACS protocols (i.e., three active and one control protocols), and is calculated as four 
161 factorial or 24 . The mean number of years of education ( \pm SD) was $16.30 \pm 3.05$ (range

from 12 to 22.5 years). Before entering the study, the participants were informed about possible adverse effects of tACS, and all of them gave their written informed consent. The exclusion criteria were history or presence of current medical, neurological or psychiatric illnesses including epilepsy, drug and/or alcohol addiction and the presence of metal implants in the head, neck and chest. In addition, the participants were examined by neurologists at the Department of Clinical Neurophysiology, University Medical Center Göttingen. The study neurologist evaluated whether any of the exclusion criteria were met. None of the participants reported any neurological or psychiatric disorders, drug-dependency, or medication acting on the central nervous system prior to or during the experiment.

\subsection{Code accessibility, data availability and ethic statement}

The Ethics Committee of the University Medical Center Göttingen approved the study, the study protocols, and all methods used therein. We performed the study in accordance with relevant guidelines and regulations. The study was registered under the study approval number 20/5/15. The study materials, code/software and pseudonymized raw data described in the paper is freely available online at https://github.com/ihrke/2020 cfc tacs.

\subsection{Experimental design}

The study used a double-blind, within-subject design. The participants underwent five experimental sessions, starting with an initial training session to familiarize themselves with the behavioral paradigm. During the training session, the participants received no 
stimulation. This initial session was followed by the four tACS sessions, the order of which was counterbalanced across participants to reduce between-session learning effects. Of the four stimulation sessions, three employed the main stimulation protocols and one the control protocol. The inter-session interval between the stimulation sessions was at least 48 hours.

\subsection{Behavioral Paradigm}

The behavioral paradigm consisted of a learning phase and a subsequent transfer phase, which was adapted from Cavanagh and colleagues (2013). The task was introduced as a card game for the participants (Figure 1). Stimuli presentation was controlled by PsychoPy (version number: 1.83.01), a free, open-source application built on the Python programming language (Peirce, 2007, 2009). For the presentation of the behavioral paradigm, we used a Dell computer with Windows 7 Enterprise 64 bit operating system, Intel (R) core i3-3220, 3.30 GHz and 4 GB RAM and a 21.5 inch Dell screen with a $1920 \times 1080$ resolution and $60 \mathrm{~Hz}$ refresh rate.

During the learning phase the participants performed a Go/NoGo instrumental learning task. Here, they had to learn action (two levels: Go/NoGo) and monetary outcome (three levels: win, no win/lose or lose) contingencies. For each card, the goal was to find the better of the two possible action choices $(\mathrm{Go} / \mathrm{NoGo})$ resulting in the highest monetary outcome (getting reward or avoiding losing) and therefore maximize their earnings.

One key feature of the task was that the action choices and monetary outcomes were orthogonal. As such, the four unique cards covered all the combinations between actions choices and monetary outcomes ('Go to win', 'NoGo to avoid losing', 'Go to avoid 
losing' and 'NoGo to win'). Due to the Pavlovian bias, i.e., approach to appetitive and withdrawal from aversive stimuli, the cards could be split into congruent and conflicting cards. For the Pavlovian congruent cards (henceforth the congruent cards; 'Go to win' and 'NoGo to avoid losing') the action selection under the automatic, Pavlovian bias was advantageous for the participants and hence easier to learn. For the Pavlovian conflicting cards (henceforth the conflicting cards; 'NoGo to win' and 'Go to avoid losing'), the action selection under the automatic, Pavlovian bias was disadvantageous for the participants and therefore harder to learn (Guitart-Masip et al., 2012).

The action outcomes were probabilistic such that $65 \%$ of correct responses led to a better outcome: Neutral monetary outcomes (no loss) for the lose cards and monetary reward for the win cards. Consequently, $35 \%$ of the correct responses led to neutral monetary outcomes for the win cards and monetary loss for the lose cards. On the other hand, wrong responses inverted this ratio, i.e. $65 \%$ of incorrect responses led to neutral monetary outcomes for the win cards and monetary loss for the lose cards. Previous studies used $80 \%$ vs. $20 \%$ or $70 \%$ vs. $30 \%$ action-outcome contingencies, which renders the present version of the probabilistic learning task slightly more difficult compared with previous versions (Cavanagh et al., 2013; Csifcsák et al., 2020; GuitartMasip et al., 2012).

For illustrative purposes, we describe possible action-outcome scenarios. Suppose card A was a 'Go to Win' card, a fact unknown to the participant. In case the participant decided to take the card, there was a 0.65 probability to receive the feedback indicating monetary reward. Consequently, there was a 0.35 probability to receive no reward. In case the participant did not take the 'Go to Win' card, the feedback probabilities were 
reversed. That is, the probability of receiving monetary reward was 0.35 and the probability of receiving no reward was 0.65

Each card was presented 20 times in a random order. Independent sets of five cards were used and randomly chosen for each session from a pool of six sets of cards. We created six card sets for the scenario that one session has to be repeated. Therefore, participants performed 80 trials in each session (20 trials $\times$ four cards) and 400 trials in total ( 80 trials $\times$ five sessions).

The presentation of the stimuli was performed in full screen mode. We set the background color of the screen to white. At the beginning of each trial, a black fixation cross (10 or $11 \mathrm{~s}$ ) was presented (see Figure 1A, Trial flow). Note that we used a relatively long duration of fixation cross in the present study compared with previous studies (e.g., Guitart-Masip et al., 2012). Also, during this time the participants were instructed to blink and swallow. This was a necessary step to increase the comparability of the present results with our other experiments using pre-stimulus intermittent tACS (manuscript in preparation) and scalp electroencephalogram recordings.

Then a card cue $(1 \mathrm{~s}$; original image size $199 \times 279$ pixels, presentation size $0.3 \times$ 0.5) was presented to the participants. We used white cards and distinguished them with a black capital letter (B, C, D, F, G, H, J, K, R, S, T, V, A, E, O, U, L, M, P, Q, W, X, Y, Z) printed in the middle of the card (see Figure 1A, Trial flow). We decorated the cards by adding four pieces of simple shapes around the letter. We used rhombus, circle and rectangle shapes and filled them with blue, gray, green, pink, orange or yellow colors. In each set, we used the same shape and color for each card.

The target detection stimulus (black circle; original image size $225 \times 220$ pixels, presentation size $0.35 \times 0.45$ ) was shown until a response occurred, or $1 \mathrm{~s}$ passed. The 
target detection stimulus indicated to the participants that they could take the card (Go) or not (NoGo), upon which the monetary outcome depended. The feedback was displayed (original image size $402 \times 205$ pixels, presentation size $0.6 \times 0.4$ ) for $2 \mathrm{~s}$ : A green ' $+€$ ' sign indicated a monetary reward, a red ' $€ €$ ' symbol indicated a monetary loss and a black horizontal bar indicated neutral monetary outcome (neither win nor loss). The next trial started $0.5 \mathrm{~s}$ after feedback.

In the subsequent transfer phase of the task (Figure 1B), the participants performed a two-alternative, forced-choice (2AFC) task where each card from the learning phase was paired with one of the three other cards following the order (e.g., 'Go to win' vs. 'NoGo to avoid losing', 'NoGo to avoid losing' vs. 'Go to win', etc.). Each of the 12 card pairs was presented four times until a response occurred, or $3 \mathrm{~s}$ passed.

The dependent variable in this study was accuracy. We defined accuracy as choosing the response category (Go/NoGo) that led with a higher probability to the better monetary outcome; hence, monetary reward for the win cards and neutral monetary outcome for the losing cards.

The participants were paid 8 EUR/hour and received an additional performance dependent bonus of 12 EUR if their mean performance calculated over all sessions was above $75 \%$. We used the monetary bonus to encourage our participants to perform as well as possible in each session. Unknown to the participants, everybody received the monetary bonus at the end of the experiment. 
A Learning phase

Pavlovian congruent
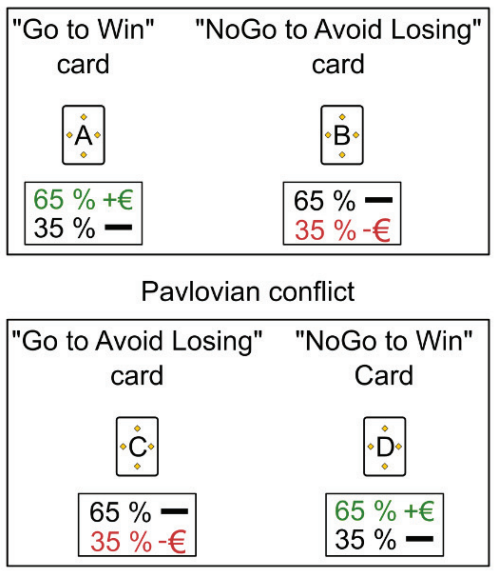

277

278

Figure 1. The structure and the trial flow of the behavioral paradigm for the learning $(A)$ and the transfer phase $(B)$.

\subsection{Transcranial alternating current stimulation}

The stimulation was delivered by a CE-certified NeuroConn® multichannel stimulator (neuroConn $\mathrm{GmbH}$, Ilmenau, Germany) during the learning phase of the task. The electrode positions were chosen according to the international 10-20 EEG system. The electrode montage was centered over the Fpz electrode location with three return electrodes positioned over the Cz, F10 and F9 positions (Figure 2A).

The following standardized steps ensured minimal stimulation-induced cutaneous sensations. After determining the electrode locations, the corresponding skin surface was gently cleaned with OneStep $®$ abrasive gel $(H+H$ Medizinprodukte $G b R$, Germany), which was removed with $0.9 \%$ saline solution (B. Braun Melsungen AG, Germany). After removing the residual saline solution with paper tissue a local anesthetic cream (Anesderm®, Pierre Fabre Dermo-Kosmetic GmbH, Germany) was 
applied for $20 \mathrm{~min}$ to numb the skin (25 mg/g lidocaine, $25 \mathrm{mg} / \mathrm{g}$ prilocaine). It was wiped off first with paper tissue followed by a skin antiseptic spray (Kodan Tinktur Forte $\circledast$, Schuelke \& Mayr GmbH, Germany). The latter was necessary to remove the anesthetic cream, which would otherwise prevent the conductive paste from adhering to the skin. Homogenous layers of Ten20® conductive paste (Waever and Company, Colorado, USA) were then applied to the skin and the electrode surfaces. Each of the four round, conductive rubber electrodes with $2 \mathrm{~cm}$ diameter (neuroConn $\mathrm{GmbH}$, Germany) was affixed to the head. The impedance was kept below $10 \mathrm{k} \Omega$. The maximal current density under the main electrode was $0.50 \mathrm{~mA} / \mathrm{cm}^{2}$. The electrode montage was prepared in a double-blind fashion.

We used four different CFC-tACS protocols, including amplitude-modulated CFCtACS (AM), CFC over the peak, trough and control tACS (Figure 2B). Each protocol started with a 20 s fade-in period, followed by a 20 -min stimulation with the maximum stimulation intensity, and ended with a 10 s fade-out period. The total stimulation duration was $20 \mathrm{~min}$ and $30 \mathrm{~s}$.

The protocols, peak-coupled, trough-coupled tACS and control, consisted of a $4 \mathrm{~Hz}$, $1 \mathrm{~mA}$ (=2 mA peak to peak) sinusoidal waveform coupled with a $0.6 \mathrm{~mA}(=1.2 \mathrm{~mA}$ peak to peak) $80 \mathrm{~Hz}$ sinusoidal waveform. These stimulation protocols had a maximum intensity of $1.6 \mathrm{~mA}$. In the peak-coupled tACS protocol, the short $80 \mathrm{~Hz}$ burst (50 ms) was coupled over the peak (38-88 ms) of each theta cycle. In the trough-coupled tACS protocol, the short $80 \mathrm{~Hz}$ burst was coupled over the trough (163-213 ms) of each theta tACS cycle. In the control stimulation, both waveforms were overlaid continuously. The control stimulation lacked any phase-specificity of gamma relative to theta oscillations but used a highly matched intensity range and identical stimulation duration with respect 
317 to the real protocols. The control protocol served as the reference to which we compared the effects of the three main CFC-tACS protocols.

In the AM protocol, the amplitude of the gamma frequency $(80 \mathrm{~Hz})$ was modulated by the phase of the theta frequency $(4 \mathrm{~Hz})$. In all protocols, the amplitude of the theta frequency was constant. Consequently, the AM protocol employed lower peak stimulation intensities (Figure 2C, left) compared with the remaining protocols, which led to a slightly higher electric field strength (Figure $2 \mathrm{C}$, right). However, this was a necessary step in order to match the amplitude of the envelope frequency in the amplitude modulation protocol to the amplitude of the theta frequency in the remaining protocols.

In order to estimate the magnitude of the induced electric field in the brain, we ran simulations using the free software package Simulations for Non-invasive Brain Stimulation (SimNIBS; version 3.0.2) (Thielscher et al., 2015). To this aim, we conducted electric field calculations on an anatomically realistic, six-compartment template head model (almi5.msh) available in SimNIBS. We used default conductivity values [S/m] that were set to 0.465 for the scalp, 0.01 for skull, 1.654 for cerebrospinal fluid, 0.275 for gray matter and 0.126 for the white matter. The simulation accounted for volumenormalized anisotropy in the brain. We observed peak electric field magnitudes up to 0.3 $\mathrm{mV} / \mathrm{mm}$ in the medial frontal cortex (Figure $2 \mathrm{C}$, right). 
A Electrode montage

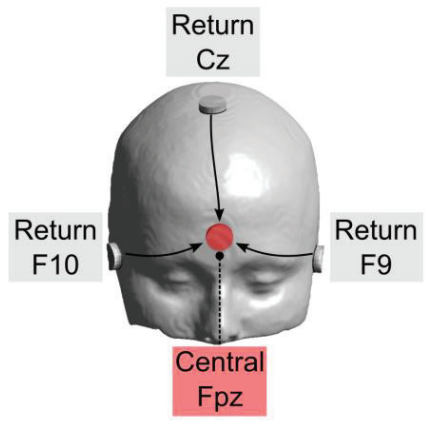

C Estimated induced electric field magnitudes
B Waveforms of CFC-tACS protocols
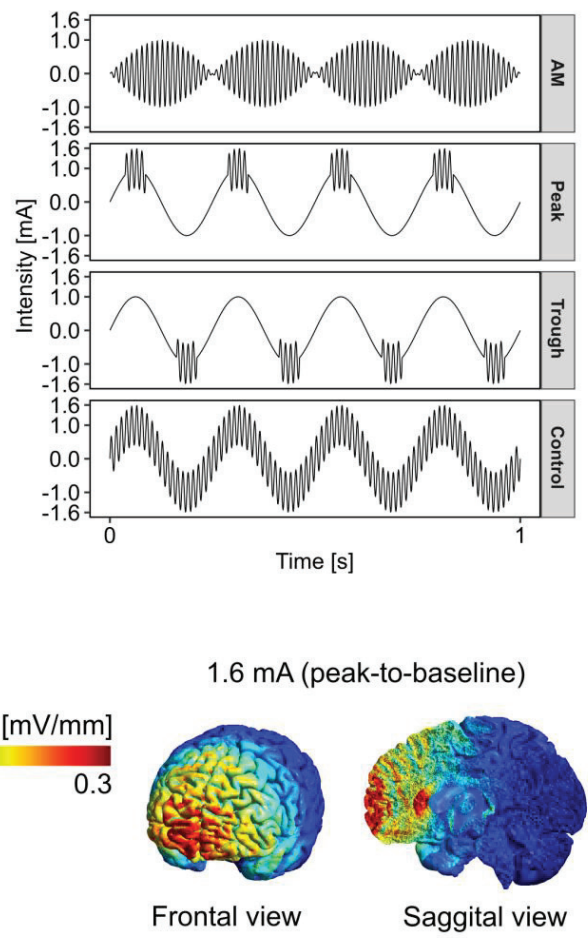

0.3

Frontal view
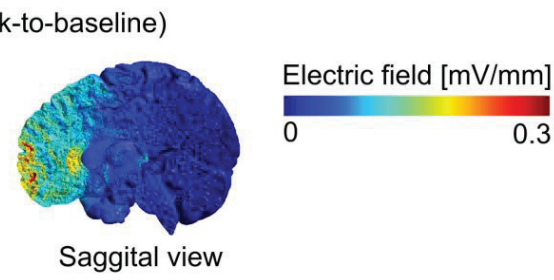

Figure 2. Stimulation parameters including electrode montage (A), cross-frequencycoupling tACS waveforms (B) and estimated electric field magnitudes in the gray matter. Electric field simulations were performed with SimNIBS version 3.0.2 on a template head model. The peak absolute electric field strength reached $0.3 \mathrm{mv} / \mathrm{mm}$ in the medial frontal cortex. Abbreviations: AM - amplitude-modulated; CFC - cross-frequency coupling.

\subsection{Procedure}

At the start of each session, the participants filled out a short questionnaire. We asked our participants to report the quality of sleep during the previous night. Further, we assessed the level of arousal ("How are you feeling right now?") with a 10-point Likert-scale where value 1 corresponded to very tired and 10 to completely awake. We 
also assessed the presence and intensity of headache ("Do you have a headache right now?") with an initial yes-no answer and an optional 10-point Likert-scale for yes responses. Here, value 1 corresponded to low and 10 to very strong headache. We assessed the intake of medication, coffee or alcohol consumption in the 24 hours before the session. The purpose of these assessments was to avoid the possibility that irregular sleep patterns in the previous night, headache or mental fatigue due to alcohol would corrupt the possible behavioral findings of tACS. Theoretically, a new session was going to be scheduled if the participant had consumed more than two alcoholic beverages in the previous day, however, arranging a new session was not necessary.

All participants received detailed written instructions about the task. Before the training session, we asked them to perform a practice session to familiarize themselves with the task and to ensure that they were able to operate the response box (RB-740, Cedrus Corporation, USA) comfortably. We used an independent set of cards in the practice session. Before the start of the learning task, the participants filled out a questionnaire to ensure that they understood the tasks correctly. The questionnaire assessed whether the participants understood i) the meaning of the three feedback types (win, no win/no loss, loss) and ii) the probabilistic nature of the feedback. In the following stimulation sessions, the short questionnaire was followed by the electrode preparation, the application of the topical anesthetic cream, and the impedance measurements. This preparation phase took approx. 35-40 minutes during which the participants watched documentary movies to maintain their vigilance.

Following the preparatory phase, the participants performed two short practice tasks. The practice tasks contained 16 trials for the learning and 12 trials for the transfer phase. 
Following the practice task and directly before the start of the learning task, the data collector opened the sealed envelope containing the information about that day's stimulation condition. After opening the envelope, the data collector selected the protocol on the stimulator and informed the participants about the start of the stimulation. Following this moment, the data collector initiated no further communication. The learning phase began directly after the fade-in period. After the end of learning phase and following a 5-minute break, the participants completed the transfer phase of the task, during which no stimulation was applied.

At the end of each session, we assessed the level of self-reported arousal, the presence and intensity of headache and secondary perceptual adverse effects associated with the application of tACS. We focused on cutaneous (i.e., itching, tingling and burning) and visual flickering sensations (i.e., phosphenes). First, the participants were asked to indicate the presence of secondary adverse effects (yes or no question). In case of a positive answer, we assessed the subjective level of discomfort using a 10point Likert scale. On the Likert scale, "1" indicated the lowest noticeable discomfort and "10" indicated an amount of discomfort the participants would not be able to endure during the experiment. The participants were informed that they could discontinue the study at any time without having to give any reason for terminating the study.

At the end of each session, we asked our participants to recall the card types and provide an internal ranking of the cards. We focused on whether the participants were able to correctly recall the cards' valence-action contingency. 


\subsection{Statistical analysis}

All statistical analyses were performed using the $\mathrm{R}$ statistical programming environment (version 3.5.1) and the RStudio integrated development environment (version 1.1.456) (R Core Team, 2018; R Studio Team, 2016). For the data analysis, we used a Precision 7920 Rack computer, Debian GNU/Linux 9.9 operating system, $2 \times$ Intel Gold 6152, 2.1GHz, 22 cores and 512 GB RAM.

We applied Bayesian methods, and we report our results in terms of the mean of the posterior distribution and their associated 95\% highest-density intervals (HDIs). These intervals are derived from the posterior distribution of the model-parameters or a combination of parameters (e.g., differences) by finding the interval that contains $95 \%$ of the posterior mass while also satisfying the criterion that all points within the interval have a higher probability density than points outside the interval (Kruschke, 2014). The interpretation of the Bayesian 95\% HDI is that it gives the range in which the estimated parameter is located with a probability of 0.95 . We consider effects to be statistically reliable, if the $95 \% \mathrm{HDI}$ excludes zero.

In order to model accuracy on the single-trial level, a dichotomous dependent variable, we used hierarchical Bayesian logistic regression. For these regression analyses, we used the R package brms (Bayesian Regression Models using Stan; Bürkner, 2018) with default, uniform priors for all regression coefficients. This package uses Hamiltonian Monte-Carlo (HMC) techniques implemented in Stan (Carpenter et al., 2017 ) to fit the models. We used four chains, where each chain had a warm-up period of 1,000 samples and 1,000 post warm-up samples resulting in a total of 4,000 posterior samples. We used the Gelman-Rubin diagnostic (Gelman \& Rubin, 1992) to ensure that all reported results had an $R \leq 1.05$. For model comparison, we used the Leave-One- 
Out Information Criterion (LOOIC), where lower scores of the LOOIC suggest a better model fit (Vehtari et al., 2017). Specifically, a model was considered better if the LOOIC score were lower, and if the $\triangle \mathrm{LOOIC}$ score were at least double the corresponding LOOIC standard error.

\subsection{Computational modelling}

The orthogonal Go-NoGo task used in our study usually allows one to fit computational reinforcement learning $(R L)$ models to the data collected during the experiment (e.g., Cavanagh \& Frank, 2014; Csifcsák et al., 2020). These models assume that each time a certain stimulus is encountered, an internal value representation of the stimulus-action pair (known as Q-value) is updated according to the reward received after taking an (in-) action. Furthermore, the decision on which action to take is based on this internal value-representation, and thus, as the Q-value gets close to the actual value with repeated encounters of a stimulus, performance becomes more accurate. The orthogonalized nature of the Go-NoGo task typically also allows the estimation of Pavlovian influences on this RL process by biasing Goresponses for rewarding stimuli and NoGo-responses for punished stimuli. We used Bayesian hierarchical modeling to fit a series of these models to our data using a strategy identical to that presented in Csifcsák and colleagues (2020), and we refer the reader to this paper and the data-repository for this paper at https://github.com/ihrke/2020 cfc tacs for technical details of the RL model. The modelcode was based on a the hBayesDM toolbox (Ahn et al., 2017).

The described computational models were implemented using the R-package rstan (Stan Development Team, 2018). We used eight parallel chains with a total of 8,000 
post-warm up samples from the posterior distribution. The convergence diagnostics were identical with the other models as described above.

\section{Results}

\subsection{Computational modeling}

We fitted models of increasing complexity to the data from our experiment. First, we fitted a model without any session-specific terms (null-model) as a baseline. Next, we modeled separate learning-rates $\alpha$, temperature parameters $\beta$, Pavlovian bias parameters $\pi$ and go-biases $b$ for each of the tACS sessions (tACS-model). Furthermore, we included a model that let each of the four core-parameters depend on the session order (order-model) and, finally, a model where separate parameters were fit for each tACS session and each parameter depended on session-order (full model).

Diagnostics of the HMC chains indicated that all models converged successfully.

We calculated the LOOIC for each of these models (see Table 1). Even though the model that only modeled the RL parameters as a function of session order received the lowest LOOIC, the differences between all four models were small compared to their standard errors (see Table 1) and model selection was therefore inconclusive. We conducted posterior predictive checks and simulated 1,000 random datasets from the posterior distribution of the parameters. Unfortunately, while some general characteristics of our participants' performance was captured by the model, it failed to properly account for the complex changes across sessions, trials and card types. Given that the computational models were unable to capture our participants' behavior, we chose not to interpret or report changes in model parameters across sessions but to focus on the more descriptive logistic regression models reported below. The reason for 
472

our failure to model our participants' performance with these established models is puzzling and deserves further investigation.

Table 1. Results of the model selection procedure for the computational models. All differences in LOOIC are small compared to their standard errors and model selection is therefore inconclusive.

\begin{tabular}{lccc} 
Model & LOOIC & $\Delta$ LOOIC & SE(LLOOIC) \\
\hline Order & 10598.3 & - & - \\
\hline Full & 10607.8 & 9.6 & 30.2 \\
\hline tACS & 10608.5 & 10.2 & 39.4 \\
\hline Null & 10615.3 & 17.0 & 33.2 \\
\hline
\end{tabular}

\subsection{Accuracy and learning}

To assess learning performance across sessions, we fitted a series of hierarchical Bayesian logistic regression models, treating accuracy as the dependent variable. All of the models received a random intercept for each participant and for sessions nested within participants. Furthermore, we included various combinations of the following predictor variables: Card-type (four levels: Go-to-Win, NoGo-to-Avoid, Go-to-Avoid and NoGo-to-Avoid), tACS session (five levels: Training, Control, AM, Peak and Trough), Trial (Z-transformed trial number during each experimental session), Session order (continuous predictor coding for the order in which the tACS sessions were conducted) as well as their interactions. All of these 20 models were compared according to their 
out-of-sample predictive performance using the LOOIC (Vehtari et al., 2017). Based on this criterion, we calculated model weights using two different techniques: Based on Akaike weights (Wagenmakers \& Farrell, 2004) using the LOOIC instead of the AIC and using Bayesian model averaging (BMA; Yao et al., 2018). Both of these techniques resulted in posterior probabilities quantifying how likely it is that each of the models was the best one.

After calculating these model selection criteria, we found converging evidence that the model that encompassed all predictors, including all two-way and three-way interactions between Card, tACS session and Trial, as well as a main effect of Session order outperformed the other models (Akaike weight $p=0.63$, next best model $p=0.34$; BMA weight $p=0.47$, next best model $p=0.23$ ).

We therefore based our conclusions on that winning model and investigated it in detail. First, we checked that the model captured the trends in the data well. In Figure 3, we plotted the raw data and overlaid predictions from the winning logistic regression model (posterior predictive check). The model captured the trends in the data well and the uncertainty (95\% HDIs) around the model-predictions was sufficiently broad relative to the fluctuations in the data. The Bayesian $R^{2}$ value for this model was $R^{2}=0.23$ $\mathrm{HDI}[0.22,0.24]$. 
504
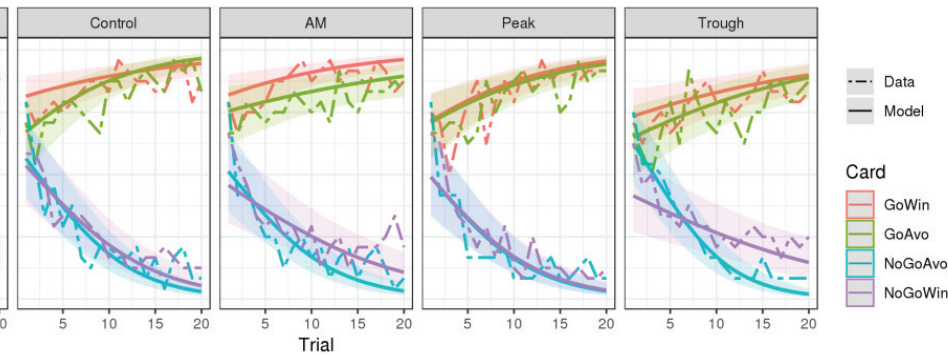

Figure 3. Posterior predictive checks for the final logistic regression model. The model predictions (solid lines) captured the main trends in the data (dashed lines) well. Colored ribbons are 95\% HDIs. Abbreviations: AM - amplitude-modulated.

We focused on two separate aspects of the data: First, we investigated how the general accuracy level varied across cards and sessions. In the presence of the threeway interaction of Card $\times$ tACS session $\times$ Trial, we quantified and compared the accuracy level in the middle of each session. Second, we were interested in the learning rate with which accurate responding increased. In our model, this was manifested in the tACS session $\times$ Trial, Card $\times$ Trial and Card $\times$ tACS session $\times$ Trial interactions that allowed us to investigate the rate with which the correct way to respond to each of the cards was learned across the sessions.

\subsection{Average accuracy}

The accuracy levels as estimated by the model in the middle of each session are displayed in Figure 4. There was a significant amount of variation both between the cards and sessions. As expected, responses to the Go-to-Win card were generally most accurate $\left(b_{\text {GoAvo }}=-.88[-1.24,-0.54], b_{\text {NoGoAvo }}=-1.02[-1.39,-0.68], b_{\text {NoGoWin }}=\right.$ 
Figure 4. Estimated accuracy levels in the middle of the experimental session for each session and card. The colors represent the four card types, with the experimental sessions shown on the horizontal axis. Note that the participants received no tACS during the training session. Abbreviations: AM - amplitude-modulated tACS; control control tACS.

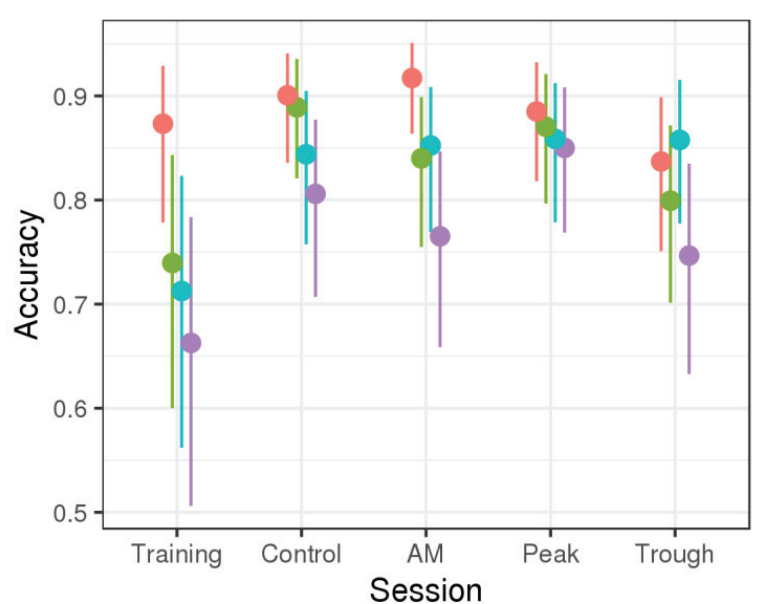

Card

GoWin

- GoAvo

1 NoGoAvo

, NoGoWin 
We were interested in how general accuracy changed between the different tACS sessions. A summary of the results is presented in Figure 5, upper row. Here, each entry in the matrix documents the posterior probability that accuracy was increased from one session (A) to the next (B). High values close to 1 (red) indicate that session A was highly likely to show increased accuracy relative to session B while low values close to zero indicate the opposite. Intermediate values (grey) mean that the results are inconclusive for that particular comparison. For example, in the 'Go to win' card the value of 0.95 in the middle row, right column suggests that it is highly probable that the average accuracy was higher in the control tACS (session A) compared to the troughcoupled tACS (session B).

We start by comparing the three active tACS sessions AM, Peak and Trough with the Control session. The $p$-values given here represent the posterior probability that the active session showed higher accuracy compared with the Control session (i.e., the probability that the difference $b$ is positive) and are not to be confused with frequentist $p$ values.

There was no clear difference between the AM and the Control session for congruent cards (GoWin: $b=0.21[-0.47,0.87], p=0.73$, NoGoAvo: $b=0.06[-0.54,0.75], p=$ 0.58 ) with possibly a small performance decrease for conflicting cards (GoAvo: $b=$ $-0.43[-1.09,0.19], p=0.10, b=-0.25[-0.85,0.38], p=0.22)$ though the HDls for these effects did not exclude zero. The Peak session did not result in a change in general accuracy compared with Control for congruent (GoWin: $b=-0.15[-0.86,0.47], p=0.32$, NoGoAvo: $b=0.11[-0.52,0.76], p=0.63$ ) or conflicting cards (GoAvo: $b=-0.19[-0.84,0.47], p=0.28$, NoGoWin: $b=0.31[-0.32,0.95], p=0.84)$. Finally, the 
557 Trough session showed reduced accuracy particularly for the easiest Go-to-Win cards,

$558 b=-0.56[-1.18,0.11], p=0.05$ (but not for NoGo-to-Avoid, $b=0.11[-0.55,0.74], p=0.62$ )

559 and reduced accuracy for both conflicting cards (GoAvo: $b=-0.70[-1.33,-0.04], p=0.02$,

560 NoGo-to-Win: $b=-0.34[-0.95,0.29], p=0.14)$. Direct comparisons between the active

561 stimulation sessions are also shown in Figure 5 (upper row).

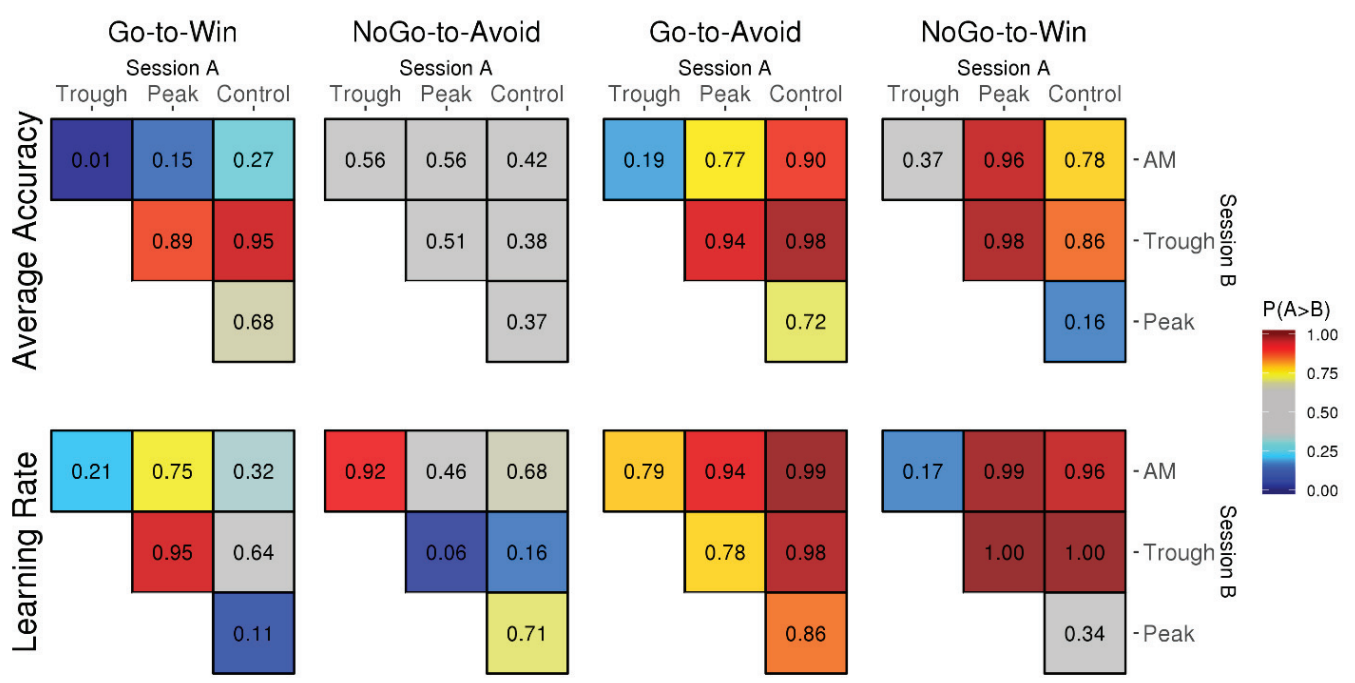

Figure 5. Comparison of average accuracy (top row) and learning rate (bottom row) between tACS sessions for each of the four cards. Colors and numbers in the matrices indicate the probability that the session indicated by the column showed a stronger effect compared with the session indicated by the row of each matrix. Abbreviations: AM - amplitude-modulated.

\subsection{Learning rate analysis}

Next, we supplemented the analysis of the general accuracy with a parallel analysis regarding the learning rate, i.e., Card and tACS session interactions with the Trial term in the model. In Figure 5 the lower row shows a summary of this analysis. AM and 
Control sessions did not differ clearly for congruent cards (GoWin:

$b=0.10[-0.30,0.52], p=0.68$, NoGoAvo: $b=-0.09[-0.47,0.31], p=0.32)$, but learning was decreased for conflicting cards (GoAvo: $b=-0.50[-0.88,-0.13], p=0.01$, NoGoWin: $b=-0.33[-0.69,0.02], p=0.04)$. For the Peak session, the results are similar but less clear, with a possible small improvement for Go-to-Win cards $(b=$ $0.24[-0.14,0.58], p=0.89)$ but not NoGo-to-Avoid $(b=-0.11[-0.51,0.28], p=0.29)$ and possibly a weak decrease for Go-to-Avoid cards $(b=-0.21[-0.61,0.18], p=0.14)$ but not for the NoGo-to-Win cards $(b=0.08[-0.30,0.44], p=0.66)$. For the Trough session, we found no clear differences for congruent cards (GoWin: $b=-0.07[-0.41,0.31], p=$ 0.36 , NoGoAvo: $b=0.21[-0.22,0.59], p=0.84$ ) but clear learning decreases for the conflicting cards (GoAvo: $b=0.36[-0.01,0.74], p=0.02$, NoGoWin:

$b=0.49[0.11,0.80], p=0.00)$.

\subsection{Perceptual adverse effects}

Most participants reported no cutaneous sensations during tACS, possibly due to the application of the topical anesthetic cream. However, we also inspected the amount of perceptual adverse effects, such as itching, tingling, and burning sensations, and phosphenes that were reported following each tACS session. A careful inspection of the subjectively reported perceptual adverse effects did not reveal any substantial differences between the stimulation sessions.

\section{Discussion}

In this study, we investigated the behavioral effects of three active theta-gamma CFC-tACS protocols in a cognitive control task. In the peak- and trough-coupled tACS 
conditions, we coupled the short bursts of $80 \mathrm{~Hz}$ gamma tACS to the local maximum, i.e., peak, or minimum, i.e., trough, of the $4 \mathrm{~Hz}$ theta tACS. In the amplitude-modulated tACS condition, we modulated the amplitude of the $80 \mathrm{~Hz}$ gamma tACS by the phase of the $4 \mathrm{~Hz}$ theta tACS. In the fourth condition, which served as a control, we continuously coupled the $80 \mathrm{~Hz}$ gamma tACS to the $4 \mathrm{~Hz}$ theta tACS.

As we had hypothesized, we found that the trough-coupled tACS condition impaired behavioral performance, in particular in the more challenging, conflicting trials. We speculate that this protocol likely interfered with the phase-dependent theta-gamma coupling between the cingulate (e.g., ACC) and the prefrontal cortices (e.g., DLPFC) (Smith et al., 2015). In a previous study using a Stroop-like interference task, information transfer analysis (Granger causality) showed that the feedback-related information travels from the ACC to the DLPFC in the theta band (Smith et al., 2015). These findings may suggest that the ACC presumably signals the need for cognitive control, whereas the DLPFC processes this information and influences ongoing behavior by exerting model-based behavioral control (Smith et al., 2015). Thus, the modulation of the information flow from the cingulate to prefrontal cortex via theta-gamma CFC could have impaired the model-based control in the trough-coupled tACS condition.

The observed behavioral effects in the present study may be due to the direct stimulation of the frontal and cingulate cortices or to indirect network effects. It has been shown in primates that there are monosynaptic connections between the frontal cortex, including the ventromedial prefrontal and cingulate cortices, to the subthalamic nucleus (Haynes \& Haber, 2013). This pathway is called the hyperdirect pathway, which supposedly exerts a strong top-down control on ongoing decisions: It influences whether an action is performed or not (Frank, 2006). One of the proposed functional relevancies 
of the hyperdirect pathway is to slow down the initial actions in cognitive control situations, when it is crucial to quickly evaluate the expected outcome of different behavioral alternatives (Frank, 2006). It is possible that the observed behavioral findings in the present study are due to the notion that the trough-coupled tACS condition indirectly interfered with the neural oscillation in the hyperdirect pathway.

At the same time, the trough-coupled tACS condition did not impair the average accuracy but it may even have slightly improved the learning rate in one of the congruent trials, i.e., 'NoGo to Avoid'. We note, however, that the statistical analysis provided only inconclusive evidence for the improvement effect in the learning rate. We therefore interpret this finding that the trough-coupled tACS condition had only negligible effect if any on the 'NoGo to Avoid' decisions and that the main effect of the troughcoupled tACS condition was interferential in nature.

Unexpectedly, the amplitude-modulated tACS condition slowed the learning rate for the conflicting trials, which is reminiscent of the behavioral effects of the trough-coupled tACS condition. However, its diminishing behavioral effect was less pronounced when compared with the trough-coupled tACS condition. In the amplitude-modulated tACS protocols, the slow, i.e., the theta frequency, might have played an important role in producing the cognitive effects of tACS (Minami \& Amano, 2017). As increased power of theta-range oscillations leads to better performance during cognitive conflict (Cavanagh et al., 2013), we would expect behavioral improvement under this protocol. Previous studies with single-frequency theta tACS showed beneficial behavioral effects in cognitive control tasks, including reduced reaction time or facilitated behavioral accuracy (Hsu et al., 2017; Lehr et al., 2019). 
Contrary to our expectations, we found no clear and consistent behavioral effects for the peak-coupled tACS protocol. In a previous study, Alekseichuk and colleagues (2016) observed behavioral improvement in the sensitivity index of a spatial working memory task during the peak-coupled tACS. Since the peak-coupled tACS protocol mimics the phase-specificity of theta-gamma CFC when signaling the need for cognitive control (Smith et al., 2015), we expected that it would increase the efficacy of the cingulate cortex to signal the need for cognitive control and thereby increase the degree of model based control implemented by the prefrontal cortex.

The lack of the behavioral effects could also have been due to the thorough instructional procedure we used in the present study. The exhaustive instructional procedure might have produced a ceiling effect, which could diminish the ability of the stimulation to further improve the performance of our volunteers. We expect that the peak-coupled tACS condition may improve the behavioral performance in groups of participants who do not reach the ceiling effect, e.g., in elderly participants or in individuals with mild cognitive impairment.

One of the limitations of the present study is that the computational modelling results were inconclusive given that the model was unable to capture our participants' behavior. Therefore, we can neither confirm nor falsify our third hypothesis concerning the underlying cognitive processes (i.e., Pavlovian bias parameter). We speculate that the lack of fit of our computational models could be, at least partially, due to the instructional procedure we used in this study. Specifically, our participants received very thorough instructions about the task including reading the written instruction, listening to the verbal explanation of the experimenter, performing the short practice, filling out the questionnaire about the task, and performing the training session. By this procedure, we 
initially intended to minimize the probability that the participants would misunderstand the task and make their decisions in a random fashion. However, the exhaustive instructional procedure likely affected the strategy of the participants, who performed very well on the task. In fact, even though our task was more difficult than that used in previous studies (e.g., in Cavanagh et al., 2013), the overall accuracy level in the tACS sessions was higher in our study indicating that the participants were potentially able to exploit the task structure to improve their reward rate.

Evidence exists that the task instruction can indirectly influence how humans perform an instrumental learning task. This phenomenon is known in the literature as the behavioral rule-governing effect (Doll et al., 2009). It is possible that after the instructional phase at least some participants were able to infer the correct structure of the task, even before the direct experience. This may have facilitated the learning process through the mechanism of confirmation bias (Doll et al., 2009); participants learned quickly to amplify those outcomes that were consistent with their internal model of the task and discarded the incompatible ones. Given the relatively difficult reward contingency probabilities ( 0.65 vs 0.35$)$, we expected much more exploration in the initial phase of the task (e.g., Csifcsák et al., 2020).

This argument is further supported by the results of the qualitative analysis we performed about the explicit knowledge of the card types. We found that all participants were able to correctly identify both the valence and the action value of the cards in the overwhelming majority of the cases (approx. 91\%). Occasionally, the participants made mistakes when identifying the correct action to the valence (approx. 8\%). Other error types were very rare. We interpret these findings as a further indirect support that the participants had explicit, rule-based knowledge about the structure of the task. 
By using a less thorough instructional procedure, future studies may use computational modeling (e.g., in Csifcsák et al., 2020) to explore the hidden parameters that may be influenced by the CFC-tACS protocol. Because these models assume that participants do gradually learn the expected value of the stimulus (Cavanagh et al., 2013; Csifcsák et al., 2020), we were not able to utilize them fruitfully in the present study.

Another possible limitation of the present study is the lack of a sham tACS protocol. Because real tACS can induce both cutaneous and visual perceptual adverse effects during the entire stimulation period, we preferred using a control tACS protocol, instead of a sham tACS protocol (Turi et al., 2013). The conventionally used fade-in, shortstimulation, fade-out sham protocols, may not be able to maintain effective blinding for the real intervention due to their shortness, as has been shown for transcranial direct current stimulation (Greinacher et al., 2019; Turi et al., 2019).

According to an alternative explanation, the control condition might have improved the behavioral performance to a similar extent to the peak-coupled tACS condition but slightly stronger than in the amplitude-modulated tACS condition. Given that the theta and gamma tACS were continuously superimposed in the control condition, this protocol had equal chance to improve or impair the behavioral performance. Therefore, this alternative explanation does not explain why the control stimulation would have improved, rather than impaired the performance. Second, a previous study applying a closely matched control protocol found no cognitive effect on a cued-recall task, even when comparing the cognitive performance before and after the intervention (Amador de Lara et al., 2017). Therefore, we find this alternative explanation to be less likely. 
709 Taken together, CFC-tACS protocols can extend single-frequency tACS protocols by 710 enabling the testing of CFC phenomena intrinsic to endogenous network oscillations 711 (Alekseichuk et al., 2016; Bächinger et al., 2017; Minami \& Amano, 2017). In this study, 712 we showed that trough-coupled tACS, i.e., when gamma tACS was coupled over the 713 trough of theta tACS, and amplitude-modulated tACS decreased the behavioral 714 performance and the use of cognitive control in healthy participants. These findings 715 suggest that the phase of coupling between theta and gamma frequencies may play an 716 important role in cognitive control.

718 Extended Data 1 - Extended data 1 contains all materials, pseudonymized raw data 719 and analysis scripts used in this study that are freely available at our repository. 


\section{References}

Ahn, W.-Y., Haines, N., \& Zhang, L. (2017). Revealing Neurocomputational Mechanisms of Reinforcement Learning and Decision-Making With the hBayesDM Package. Computational Psychiatry, 1(Figure 1), 24-57. https://doi.org/10.1162/cpsy_a_00002

Alekseichuk, I., Turi, Z., Amador de Lara, G., Antal, A., \& Paulus, W. (2016). Spatial Working Memory in Humans Depends on Theta and High Gamma Synchronization in the Prefrontal Cortex. Current Biology, 26(12), 1513-1521. https://doi.org/10.1016/j.cub.2016.04.035

Amador de Lara, G., Alekseichuk, I., Turi, Z., Lehr, A., Antal, A., \& Paulus, W. (2017). Perturbation of theta-gamma coupling at the temporal lobe hinders verbal declarative memory. Brain Stimulation, 1-9. https://doi.org/10.1016/j.brs.2017.12.007

Bächinger, M., Zerbi, V., Moisa, M., Polania, R., Liu, Q., Martini, D., Ruff, C., \& Wenderoth, N. (2017). Concurrent tACS-fMRI Reveals Causal Influence of Power Synchronized Neural Activity on Resting State fMRI Connectivity. The Jounral of Neuroscience, 37(18), 4766-4777. https://doi.org/10.1523/JNEUROSCI.175616.2017

Bürkner, P. C. (2018). Advanced Bayesian multilevel modeling with the R package brms. The R Journal, 10(1), 395-411. https://doi.org/10.32614/rj-2018-017 
Canolty, R. T., \& Knight, R. T. (2010). The functional role of cross-frequency coupling. Trends in Cognitive Sciences, 14(11), 506-515. https://doi.org/10.1016/j.tics.2010.09.001

Carpenter, B., Gelman, A., Hoffman, M., Lee, D., Goodrich, B., Betancourt, M., Brubaker, M. A., Li, P., \& Riddell, A. (2017). Stan : A Probabilistic Programming Language. Journal of Statistical Software, 76(1), 2-32.

Cavanagh, J. F., Eisenberg, I., Guitart-Masip, M., Huys, Q., \& Frank, M. J. (2013). Frontal Theta Overrides Pavlovian Learning Biases. Journal of Neuroscience, 33(19), 8541-8548. https://doi.org/10.1523/JNEUROSCI.5754-12.2013

Cavanagh, J. F., \& Frank, M. J. (2014). Frontal theta as a mechanism for cognitive control. Trends in Cognitive Sciences, 18(8), 414-421. https://doi.org/10.1016/j.tics.2014.04.012

Cohen, M. X. (2014). A neural microcircuit for cognitive conflict detection and signaling. Trends in Neurosciences, 37(9), 480-490. https://doi.org/10.1016/j.tins.2014.06.004

Csifcsák, G., Melsæter, E., \& Mittner, M. (2020). Intermittent Absence of Control during Reinforcement Learning Interferes with Pavlovian Bias in Action Selection. Journal of Cognitive Neuroscience, 32(4), 646-663.

Doll, B. B., Jacobs, W. J., Sanfey, A. G., \& Frank, M. J. (2009). Instructional control of reinforcement learning : A behavioral and neurocomputational investigation. Brain Research, 1299, 74-94. https://doi.org/10.1016/j.brainres.2009.07.007

Frank, M. J. (2006). Hold your horses: A dynamic computational role for the subthalamic 
nucleus in decision making. Neural Networks, 19(8), 1120-1136.

https://doi.org/10.1016/j.neunet.2006.03.006

Gelman, A., \& Rubin, D. B. (1992). Interference from iterative simulation using multiple sequences. Statistical Science, 7(4), 457-511. https://doi.org/10.1214/ss/1177013437

Greinacher, R., Buhôt, L., Möller, L., \& Learmonth, G. (2019). The time course of ineffective sham-blinding during low-intensity $(1 \mathrm{~mA})$ transcranial direct current stimulation. European Journal of Neuroscience, 50(8), 3380-3388. https://doi.org/10.1111/ejn.14497

Guitart-Masip, M., Chowdhury, R., Sharot, T., Dayan, P., Duzel, E., \& Dolan, R. J. (2012). Action controls dopaminergic enhancement of reward representations. Proceedings of the National Academy of Sciences, 109(19), 7511-7516. https://doi.org/10.1073/pnas.1202229109

Guitart-Masip, M., Duzel, E., Dolan, R., \& Dayan, P. (2014). Action versus valence in decision making. Trends in Cognitive Sciences, 18(4), 194-202. https://doi.org/10.1016/j.tics.2014.01.003

Haynes, W. I. A., \& Haber, S. N. (2013). The organization of prefrontal-subthalamic inputs in primates provides an anatomical substrate for both functional specificity and integration: Implications for basal ganglia models and deep brain stimulation. Journal of Neuroscience, 33(11), 4804-4814.

https://doi.org/10.1523/JNEUROSCI.4674-12.2013 
Helfrich, R. F., \& Knight, R. T. (2019). Cognitive neurophysiology of the prefrontal cortex. In Handbook of Clinical Neurology (1st ed., Vol. 163). Elsevier B.V. https://doi.org/10.1016/B978-0-12-804281-6.00003-3

Hsu, W. Y., Zanto, T. P., Schouwenburg, M. R. V., \& Gazzaley, A. (2017). Enhancement of multitasking performance and neural oscillations by transcranial alternating current stimulation. PlosOne, 1-17.

Kruschke, J. (2014). Doing Bayesian data analysis: A tutorial with R, JAGS, and Stan. Academic Press.

Lehr, A., Henneberg, N., Nigam, T., Paulus, W., \& Antal, A. (2019). Modulation of Conflict Processing by Theta-Range tACS over the Dorsolateral Prefrontal Cortex. Neural Plasticity.

Minami, S., \& Amano, K. (2017). Illusory Jitter Perceived at the Frequency of Alpha Report Illusory Jitter Perceived at the Frequency of Alpha Oscillations. Current Biology, 27(15), 2344-2351.e4. https://doi.org/10.1016/j.cub.2017.06.033

Negahbani, E., Kasten, H. F., Herrmann, C. S., \& Fröhlich, F. (2018). Targeting Alphaband Oscillations in a Cortical Model with Amplitude-Modulated High-Frequency Transcranial Electric Stimulation. Neuroimage, 173, 3-12. https://doi.org/10.1016/j.physbeh.2017.03.040

Peirce, J. W. (2007). PsychoPy-Psychophysics software in Python. Journal of Neuroscience Methods, 162(1-2), 8-13. https://doi.org/10.1016/j.jneumeth.2006.11.017 
Peirce, J. W. (2009). Generating stimuli for neuroscience using PsychoPy. Frontiers in Neuroinformatics, 2(10), 1-8. https://doi.org/10.3389/neuro.11.010.2008

Peterchev, A. V., Wagner, T. A., Miranda, P. C., Nitsche, M. A., Paulus, W., Lisanby, S. H., Pascual-leone, A., \& Carolina, N. (2012). Fundamentals of transcranial electric and magnetic stimulation dose: Definition, selection, and reporting practices. Brain Stimulation, 5(4), 435-453. https://doi.org/10.1016/j.brs.2011.10.001

Shenhav, A., Musslick, S., Lieder, F., Kool, W., Griffiths, T. L., Cohen, J. D., \& Botvinick, M. M. (2017). Toward a Rational and Mechanistic Account of Mental Effort. Annual Review of Neuroscience, 40, 99-124.

Smith, X. E. H., Banks, X. G. P., Mikell, C. B., Cash, X. S. S., Patel, S. R., Eskandar, E. N., \& Sheth, S. A. (2015). Frequency-Dependent Representation of ReinforcementRelated Information in the Human Medial and Lateral Prefrontal Cortex. The Journal of Neuroscience, 35(48), 15827-15836. https://doi.org/10.1523/JNEUROSCI.186415.2015

Team, R. C. (2018). R: A language and environment for statistical computing. R Foundation for Statistical Computing. https://www.r-project.org/

Team, Rs. (2016). RStudio: Integrated Development for R. RStudio, Inc., Boston, MA.

Team, S. D. (2018). RStan: The R Interface to Stan. http://mc-stan.org/

Thielscher, A., Antunes, A., \& Saturnino, G. B. (2015). Field modeling for transcranial magnetic stimulation: A useful tool to understand the physiological effects of TMS? Proceedings of the Annual International Conference of the IEEE Engineering in 
Medicine and Biology Society, EMBS, 222-225.

https://doi.org/10.1109/EMBC.2015.7318340

Turi, Z., Ambrus, G. G., Janacsek, K., Emmert, K., Hahn, L., Paulus, W., \& Antal, A. (2013). Both the cutaneous sensation and phosphene perception are modulated in a frequency-specific manner during transcranial alternating current stimulation. Restorative Neurology and Neuroscience, 31(3), 275-285. https://doi.org/10.3233/RNN-120297

Turi, Z., Opitz, A., Groot, J., Thielscher, A., \& Hawkins, G. E. (2019). Blinding is compromised for transcranial direct current stimulation at $1 \mathrm{~mA}$ for $20 \mathrm{~min}$ in young healthy adults. February, 1-8. https://doi.org/10.1111/ejn.14403

Vehtari, A., Gelman, A., \& Gabry, J. (2017). Practical Bayesian model evaluation using leave-one-out cross-validation and WAIC. Statistics and Computing, 27(5), 14131432. https://doi.org/10.1007/s11222-016-9696-4

Wagenmakers, E. J., \& Farrell, S. (2004). AIC model selection using Akaike weights. Psychonomic Bulletin and Review, 11(1), 192-196. https://doi.org/10.3758/BF03206482

Yao, Y., Vehtari, A., Simpson, D., \& Gelman, A. (2018). Using Stacking to Average Bayesian Predictive Distributions (with Discussion). Bayesian Analysis, 13(3), 9171007. https://doi.org/10.1214/17-ba1091 


\section{Authors contribution}

ZT: conceptualization, study design, project administration, methodology, software (behavioral paradigm), supervised data collection, supervised medical student, prepared illustrations, data visualization, interpreted data, data curation, wrote original draft and revised manuscript.

MM: formal statistical analysis, data visualization, computational modelling of behavioral data, interpreted data, data curation, wrote original draft and revised manuscript.

AL: contributed to formal analysis, contributed to preparing illustrations, data visualization, interpreted data and wrote original draft and revised manuscript.

HB: data collection (as part of her medical dissertation at the University Medical Center Göttingen, Germany, supervised by author AA), transcribed data, contributed to writing original draft and revised manuscript.

AA: project administration, supervised medical student, contributed to writing original draft and revised manuscript. WP: study design, resources and funding acquisition, contributed to writing original draft and revised manuscript.

\section{Acknowledgements}

The authors wish to thank Dr. med. Anja Manig, Dr. med. Sebastian Schade, Dr. med. Dirk Czesnik and Dr. med. Claire Halsband for the neurological examinations. We thank Prof. Thomas Crozier for his comments on the manuscript. 


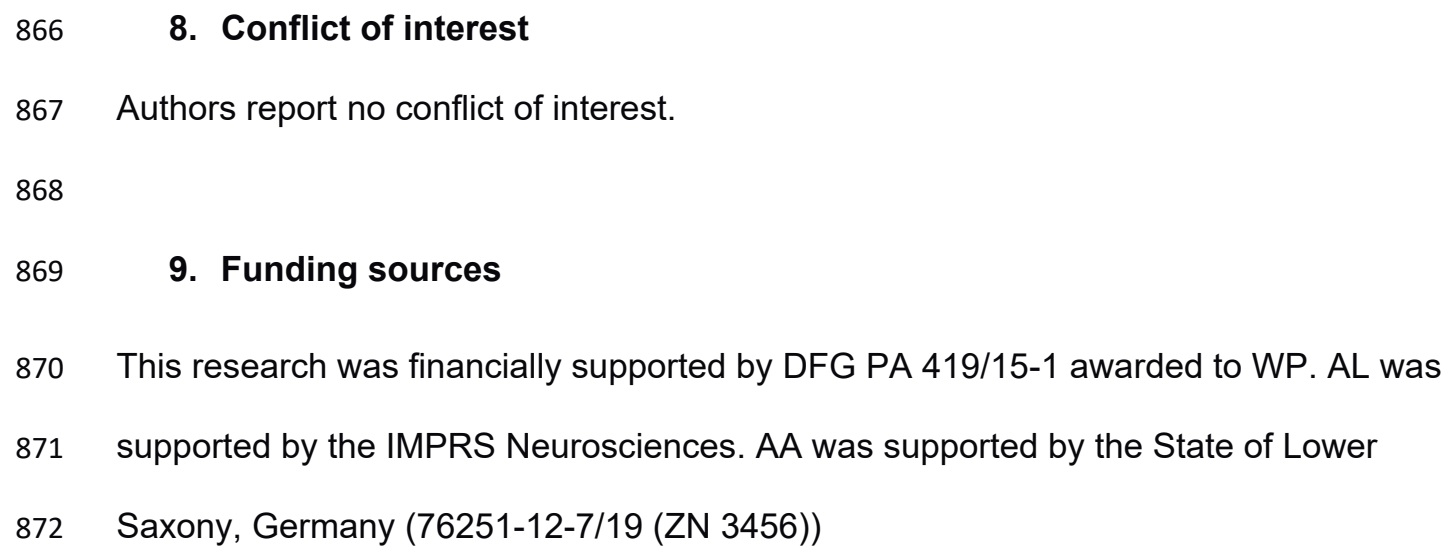


A Learning phase

Pavlovian congruent

\begin{tabular}{|c|c|}
\hline $\begin{array}{l}\text { "Go to Win" } \\
\text { card }\end{array}$ & $\begin{array}{c}\text { "NoGo to Avoid Losing" } \\
\text { card }\end{array}$ \\
\hline$\therefore \circ$ & ${ }^{\circ} B^{\circ}$ \\
\hline $\begin{array}{l}65 \%+€ \\
35 \%-\end{array}$ & 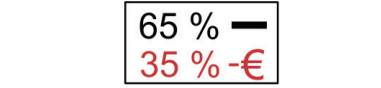 \\
\hline
\end{tabular}

Pavlovian conflict
"Go to Avoid Losing" "NoGo to Win" card

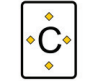

$65 \%-$

$35 \%-€$
Card

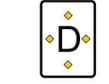

$65 \%+€$ $35 \%-$
Trial flow

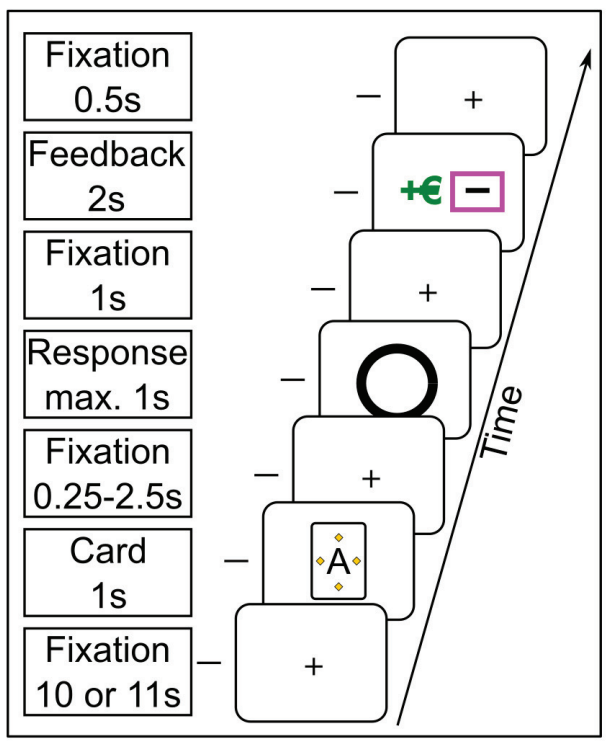

B Transfer phase

All card combinations
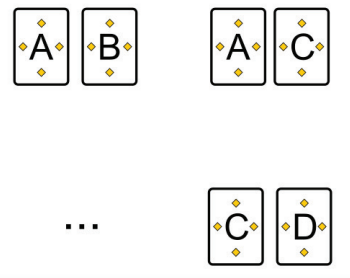

Trial flow

\begin{tabular}{|c|c|c|}
\hline $\begin{array}{c}\text { Fixation } \\
3 \text { or } 2 s \\
\end{array}$ & - & + \\
\hline $\begin{array}{c}\text { 2AFC } \\
\max .3 s\end{array}$ & $-\stackrel{A}{A} \cdot$ & $B$ \\
\hline $\begin{array}{c}\text { Fixation } \\
2 \text { or } 3 \mathrm{~s}\end{array}$ & + & \\
\hline
\end{tabular}




\section{A Electrode montage}

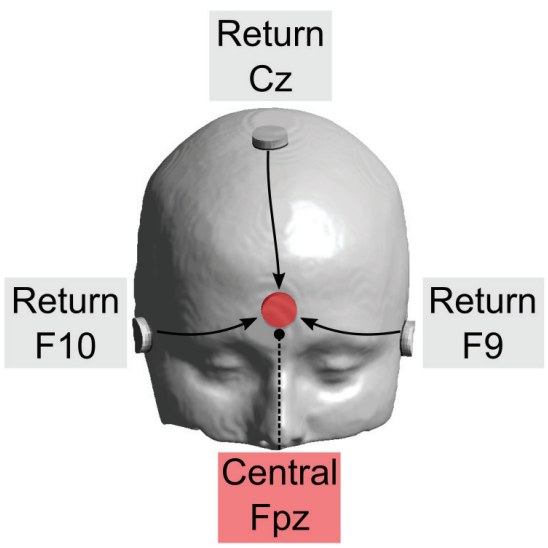

\section{Estimated induced electric field magnitudes}

\section{(d)}

$$
1 \mathrm{~mA} \text { (peak-to-baseline) }
$$

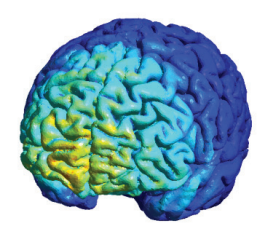

Frontal view

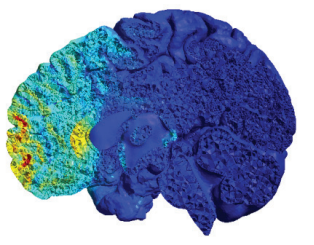

Saggital view

B Waveforms of CFC-tACS protocols

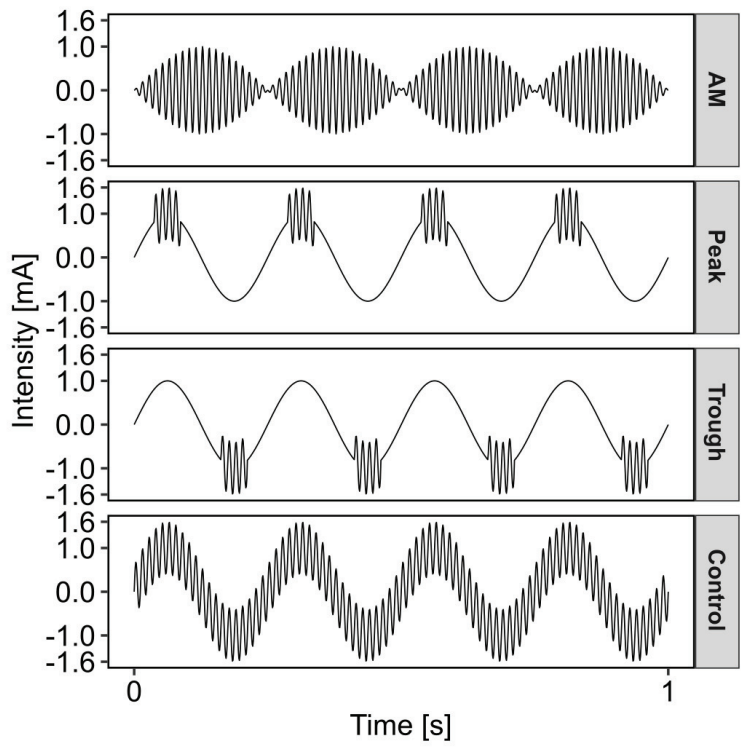

\section{$1.6 \mathrm{~mA}$ (peak-to-baseline)}

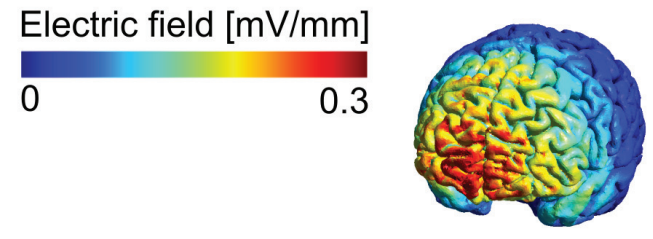

Frontal view

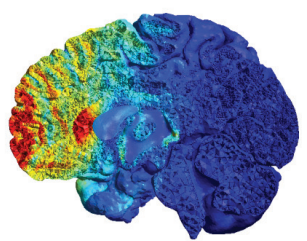

Saggital view 

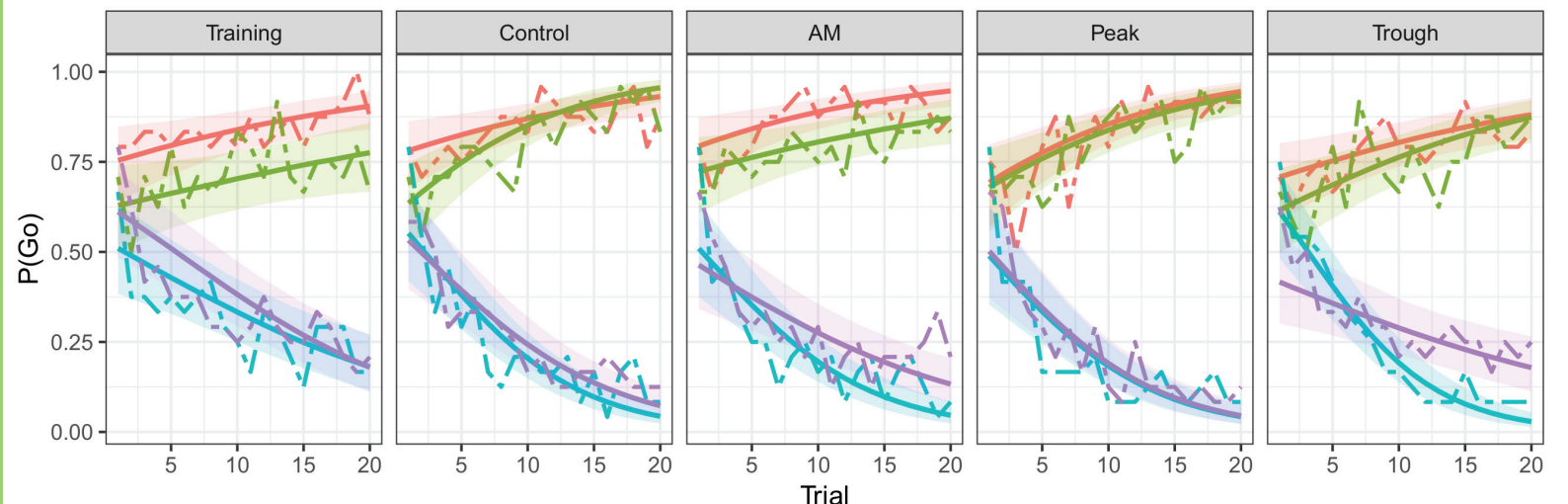

--. Data

- Model

Card

$\square$ GoWin
$\square$ GoAvo
$\square$ NoGoAvo
$\square$ NoGoWin

(U)

(U)

$\checkmark$

O

5

(U)

(1) 


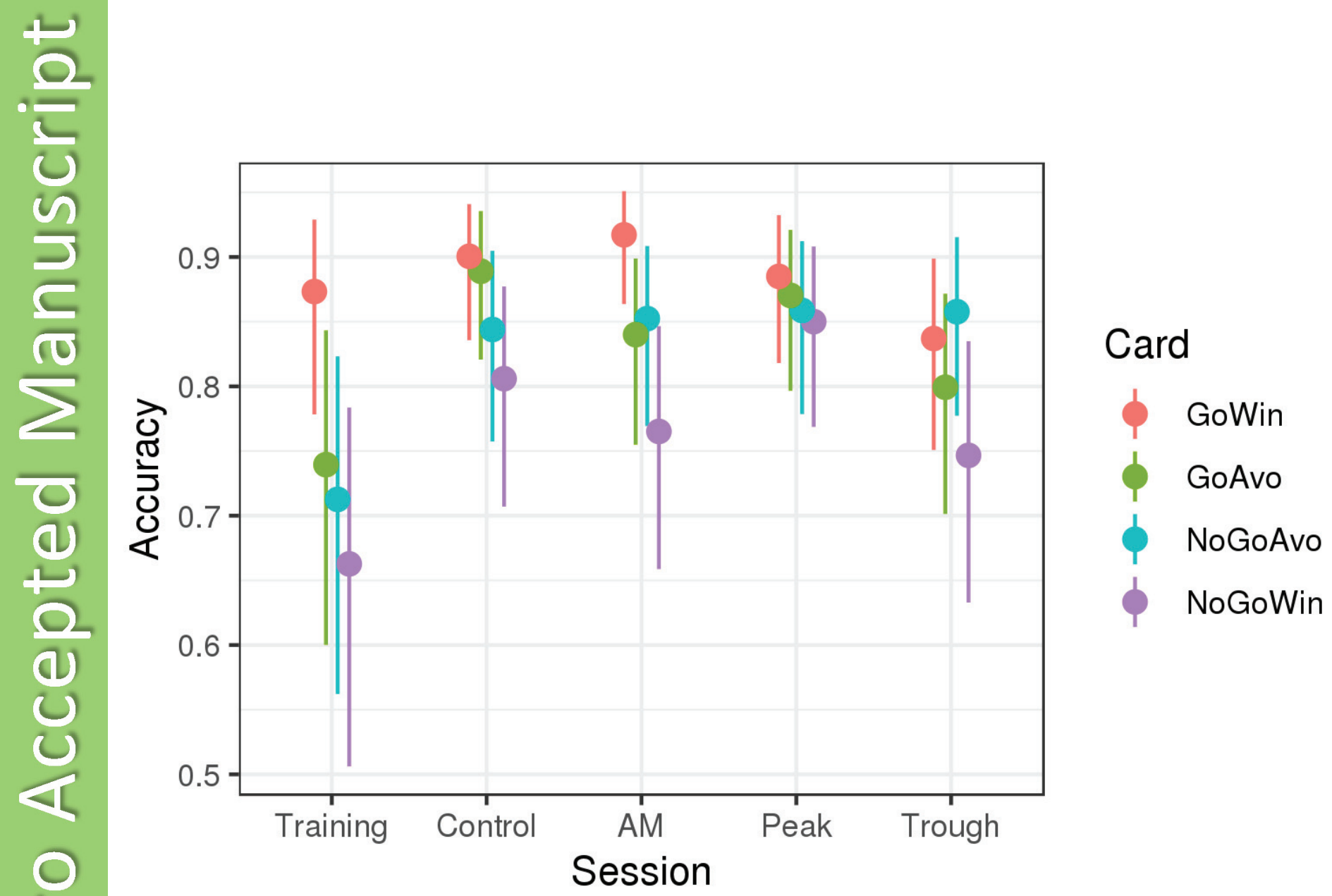




\section{Go-to-Win \\ NoGo-to-Avoid}

Trough Peak Control
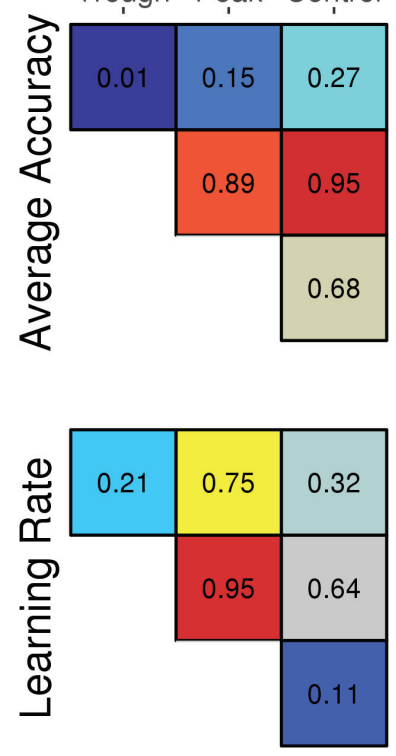

Session A

Trough Peak Control
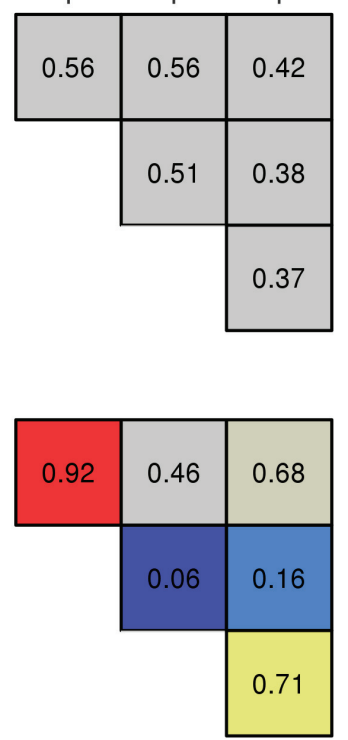

Go-to-Avoid

Session A

Trough Peak Control
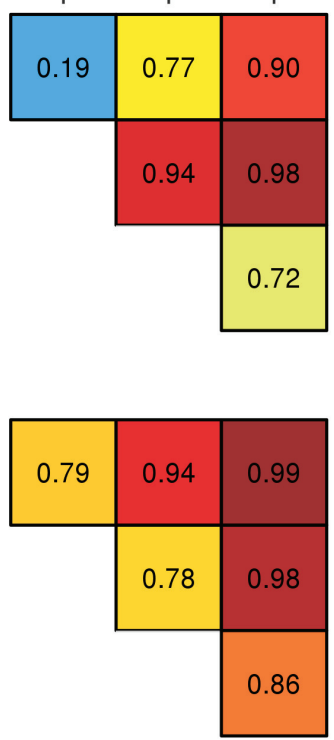

\section{NoGo-to-Win}

Session A

Trough Peak Control
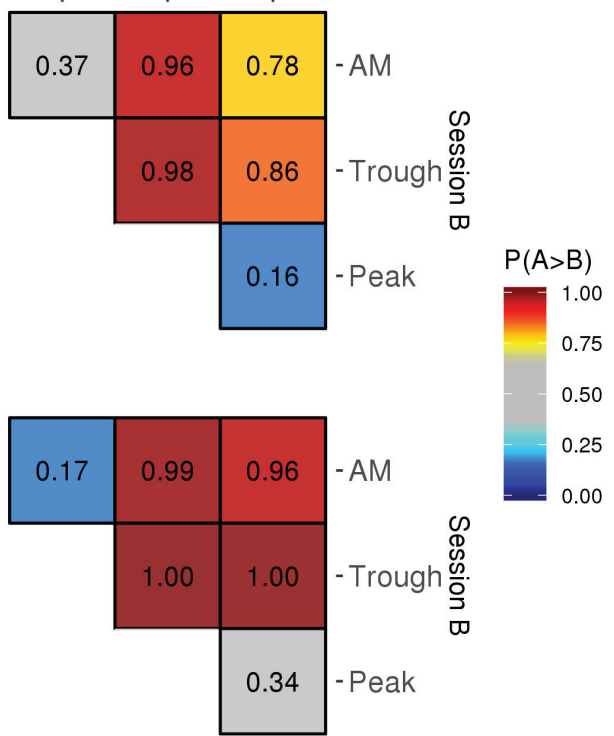